\title{
BMJ Open Chronic kidney disease as a risk factor for acute community-acquired infections in high-income countries: a systematic review
}

\author{
Helen I McDonald, ${ }^{1}$ Sara L Thomas, ${ }^{2}$ Dorothea Nitsch ${ }^{1}$
}

To cite: McDonald HI, Thomas SL, Nitsch D. Chronic kidney disease as a risk factor for acute community-acquired infections in high-income countries: a systematic review. BMJ Open 2014;4: e004100. doi:10.1136/ bmjopen-2013-004100

- Prepublication history and additional material is available online. To view please visit the journal (http://dx.doi.org/ 10.1136/bmjopen-2013004100).

Received 23 September 2013 Revised 20 February 2014 Accepted 27 February 2014

CrossMark

\begin{abstract}
${ }^{1}$ Department of Noncommunicable Disease Epidemiology, London School of Hygiene \& Tropical Medicine, London, UK ${ }^{2}$ Department of Infectious Disease Epidemiology, London School of Hygiene \& Tropical Medicine, London, UK
\end{abstract}

Correspondence to Dr Helen I McDonald; helen.mcdonald@Ishtm.ac.uk

\section{ABSTRACT}

Objective: A systematic review of the association of predialysis chronic kidney disease (CKD) with the incidence of acute, community-acquired infections. Design: We searched the MEDLINE, EMBASE and Cochrane databases (inception to 16 January 2014) for studies analysing the association of predialysis kidney disease with the incidence of acute, communityacquired urinary tract infection (UTI), lower respiratory tract or central nervous system infections or sepsis. Studies were required to include at least 30 participants with and without kidney disease.

Setting and participants: Community-based populations of adults in high-income countries. Outcome measures: Acute, community-acquired UTI, lower respiratory tract or central nervous system infections or sepsis.

Results: We identified 14 eligible studies. Estimates from two studies lacked $95 \% \mathrm{Cls}$ and SEs. The remaining 12 studies yielded 17 independent effect estimates. Only three studies included infections managed in the community. Quality assessment revealed that probable misclassification of kidney disease status and poor adjustment for confounding were common. There was evidence from a few large high-quality studies of a graded association between predialysis CKD stage and hospitalisation for infection. One study found an interaction with age, with a declining effect of CKD on infection risk as age increased. There was evidence of between-studies heterogeneity $\left(I^{2}=96.5 \%, p<0.001\right)$ which persisted in subgroup analysis, and thus meta-analysis was not performed.

Conclusions: Predialysis kidney disease appears to be associated with increased risk of severe infection. Whether predialysis kidney disease increases the susceptibility to infections and whether age modifies this association remains unclear.

\section{INTRODUCTION}

Chronic kidney disease (CKD) is common, and its prevalence is increasing. ${ }^{1}$ Infection is a major cause of mortality in end-stage renal

\section{Strengths and limitations of this study}

- This study used a sensitive search strategy, with a broad definition of kidney disease, for a thorough and inclusive search.

- Study quality was assessed using a tool adapted to observational studies, providing a transparent assessment of the risk of a range of biases for each study.

- Between-study heterogeneity and the low quality of many of the studies limit the interpretation of results of the studies currently available.

disease (ESRD) and hospitalisation at all stages of CKD. The second commonest cause of death among patients with ESRD in the USA is septicaemia, and patients with ESRD are at increased risk of death from infection compared to the general population. ${ }^{2-4}$ Patients with ESRD and predialysis CKD in the USA are at higher risk of hospitalisation for infection than the general population. ${ }^{256}$ Predialysis CKD has been found to increase mortality among patients hospitalised with infections. $^{7}$

Increased mortality and hospitalisation from infection could be driven by increased severity of infection, that is, once an infection is present, the course of the associated illness is more severe, or increased incidence, that is, CKD may make people more susceptible to develop an infection. Patients with CKD display impaired host immunity: reduced vaccination responsiveness is observed at all stages of CKD. ${ }^{8}$

Among patients with ESRD, aspects of dialysis, such as vascular and peritoneal access for dialysis, may be a risk factor for infection incidence and severity. However, this does not tell the whole story, and only $23 \%$ of infectionrelated hospitalisations among patients undergoing haemodialysis in the USA were identified as related to vascular access in the 
HEMO study. ${ }^{9}$ Risk factors for infection identified among patients with ESRD which are not related to renal replacement therapy, and could apply at all stages of predialysis CKD, include: the causes and treatment of kidney disease; comorbidities; reduced vaccine effectiveness; and high levels of exposure to healthcare facilities. ${ }^{10}$

If there is an increased risk of infection incidence at early stages of CKD, this would affect a large and growing number of patients. Awareness and quantification of this risk could have benefits for patient management, more effective vaccination strategies and healthcare planning.

Narrative reviews have concluded that it is likely that CKD in itself increases infection incidence, but reported a lack of evidence. ${ }^{10-12}$ We are not aware of any relevant systematic literature reviews of the effect of CKD on infection incidence.

This review sought to assess systematically whether predialysis CKD is a risk factor for the incidence of acute, community-acquired urinary tract infection (UTI), lower respiratory tract infection (LRTI), central nervous system (CNS) infection or sepsis, among communitybased adults in high-income countries.

\section{METHODS}

\section{Data sources and searches}

One reviewer (HIM) searched the MEDLINE and EMBASE databases, and the Cochrane library, from inception to 16 January 2014. The search strategies combined text words and MeSH terms for three concepts: acute community-acquired infection (sepsis, UTI, LRTI or CNS infection), kidney disease and relative risk. We used search terms to identify studies among adult humans in high-income countries (according to the World Bank classification), ${ }^{13}$ and limited the search to articles in English, French or German. The full strategies are available in online supplementary tables S1-S3.

We searched the reference lists of all included studies and any pertinent review articles to identify further eligible studies.

\section{Study selection}

One reviewer (HIM) screened titles and abstracts, reviewed the full text of identified studies and made initial decisions on eligibility according to prespecified inclusion criteria (see online supplementary table S4). Any borderline cases were discussed between HIM, DN and SLT. A second reviewer (DN) checked a sample of 100 abstracts, selected randomly after de-duplication of records, and a $\kappa$ statistic was calculated to describe agreement in the selection of studies.

Eligible studies analysed the effect of predialysis kidney disease on the relative risk of at least one of the four specified acute, community-acquired infections among community-based adults in high-income countries. We excluded study populations managed in secondary care (unless for kidney disease), routinely treated with immunosuppressants, or exclusively of pregnant women, as these groups have a raised risk of infection, and the relationship of CKD to infection risk may be different among these groups compared to that in the general adult population in primary care. Ascertainment of CKD, as a silent disease, and, to a certain extent, ascertainment of acute community-acquired infections are dependent on high levels of monitoring and good access to healthcare, so we restricted our search to high-income countries. Chronic infections such as tuberculosis were not included, as the relationship between CKD and chronic infection is very likely to differ from that between CKD and acute infections, which was our focus in this review.

To maximise the sensitivity of our search strategy, we accepted a wide range of definitions of kidney disease, including: medical diagnosis of kidney disease, reduced estimated glomerular filtration rate or creatinine clearance, elevated creatinine, proteinuria, microalbuminuria or macroalbuminuria and renal structural abnormalities. We also accepted definitions which included some patients with ESRD among the patients with CKD, but excluded definitions which were exclusively patients receiving renal replacement therapy.

Outcomes of interest were relative risk estimates of acute community-acquired LRTIs, UTIs, CNS infections or sepsis. We accepted outcomes describing incidence of severe infections (such as hospitalisation with pneumonia).

We restricted our search to published studies which were sufficiently large to include at least 30 participants with and without kidney disease, to allow reasonable precision of the study estimate. Detailed eligibility criteria are listed in online supplementary table S4.

\section{Data extraction and quality assessment}

Data were extracted from relevant studies using a prespecified collection form. Study characteristics extracted included study design, data source, any participant exclusion criteria, number of participants, age, gender, baseline renal function, definition of renal impairment and definition of the outcome infection. An estimate of relative risk (rate ratio, risk ratio or OR) with any measures taken to address confounding was extracted from each eligible independent analysis in each study. Studies with no CIs and for which the SE was not calculable from the data presented were included in the review but not considered for meta-analysis.

When multiple estimates were available from a study but were not independent, a single estimate was identified for potential meta-analysis by selecting the estimate best adjusted for confounding, using the most recent data, comparing the level of CKD most common in the general population with no CKD.

Study quality was assessed using a prespecified tool adapted from Higgins et $a l^{14}$ for observational studies. Studies were assigned a high, low or uncertain risk of each of the following: selection bias, non-differential measurement error for exposure and outcome, information bias in exposure and outcome, confounding and reverse causation. The minimum requirement for a low risk of bias 
from confounding was appropriate management of confounding by age, sex and diabetes. The specific criteria used are detailed in online supplementary table S5.

\section{Data synthesis and analysis}

The relationship between CKD and UTIs was considered likely to differ from that of CKD to other infections, due to potential reverse causality. For example, repeat UTIs may cause kidney disease, or structural kidney disease may be identified through investigation of repeat UTIs. Therefore, in all quantitative analysis, UTIs were analysed separately from other infections.

Estimates were examined for heterogeneity using Cochran's $Q$ statistic and the $\mathrm{I}^{2}$ statistic as described by Higgins et al. $^{15}$ If $\mathrm{I}^{2}$ was less than $50 \%$ and Cochran's $Q$ statistic $\mathrm{p} \geq 0.1$, fixed-effects meta-analysis was considered for each of the two categories (UTI and other infections). Funnel plots were constructed to look for publication bias. All analysis was conducted using STATAV.12.0.

\section{RESULTS}

The database searches identified 10380 citations, of which 1204 were duplicates (figure 1). Both reviewers had $100 \%$ agreement on which studies to extract for fulltext analysis from screening a random sample of 100 abstracts (Cohen's $\mathrm{K}=1$ ).

Figure 1 Flow chart of study selection. *Common examples of ineligible studies returned by the database searches included: studies in which renal failure and infection were both outcomes, studies in which renal failure and infection were both exclusion criteria, studies of acute renal failure resulting from sepsis or antibiotic use, studies of chronic infections (e.g. hepatitis C, BK viraemia, tuberculosis) following organ transplantation, descriptive studies of UTIs, descriptive studies of CKD, studies of predictors of prognosis among patients with infections, and review articles without any original data.
We identified 14 eligible studies, with varying study characteristics (table 1). Four studies were case-control studies, ${ }^{16-19}$ and 10 were cohort studies. ${ }^{20-29}$ Seven studies investigated a range of risk factors for infection, ${ }^{16-19} 212829$ two studies reported the effect of CKD on infection as a confounder of the effect of interest ${ }^{24} 25$ and five studies investigated the effect of CKD on infection risk as their primary research question. ${ }^{520} 222627$

Seven studies were based among the general population. $^{5} 161921232829$ Other study populations included: attendants at a specialist renal clinic, ${ }^{22}$ patients with diabetes mellitus, ${ }^{25}$ patients admitted to hospital for an acute cardiovascular event or an arterial revascularisation procedure, ${ }^{24}$ and the Navajo Nation-a population which experiences 3-5 times higher rates of invasive pneumococcal disease than the general US population. ${ }^{17}$ The population of the cohort studies in Calgary, Canada comprised adults with a serum creatinine test result available in their medical records. ${ }^{26} 27$ There is some overlap in the study populations of these two cohort studies: residents aged over 65 years with a serum creatinine measurement between 1 July 2001 and 31 December 2001 and also between 1 July 2003 and 30 June 2004 would have been included in both studies for the period from the second creatinine measurement until 31 December 2004. ${ }^{26} 27$

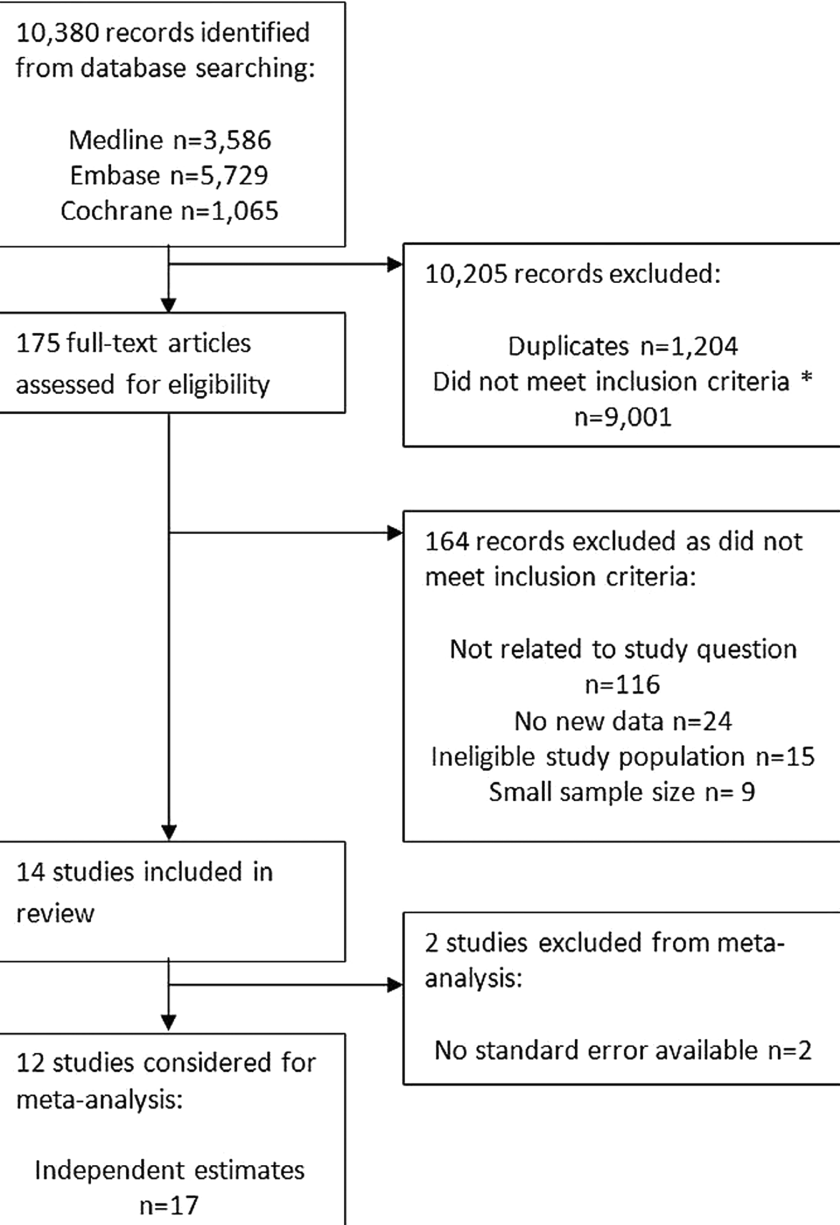


Table 1 Characteristics of eligible studies $(n=14)$

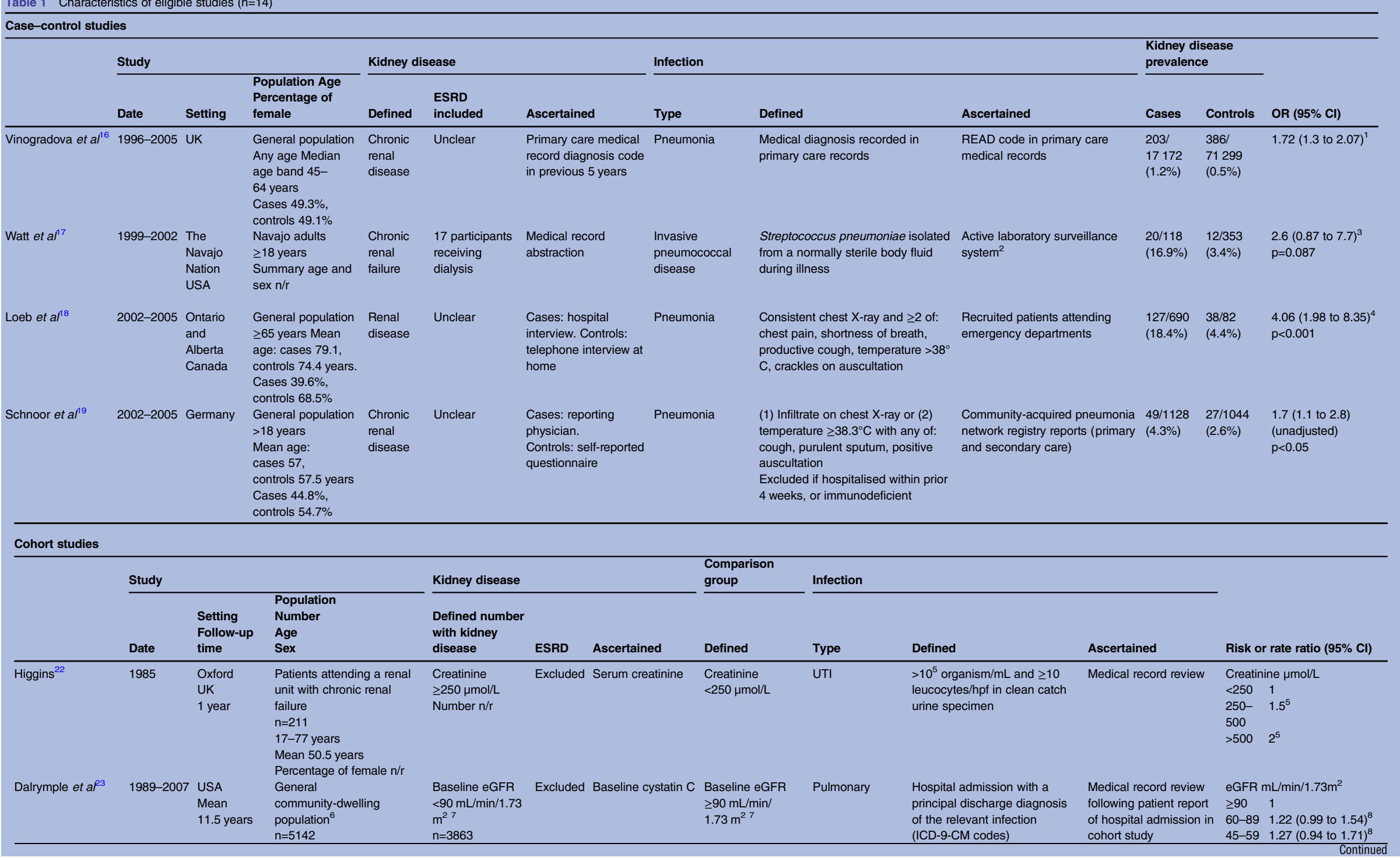




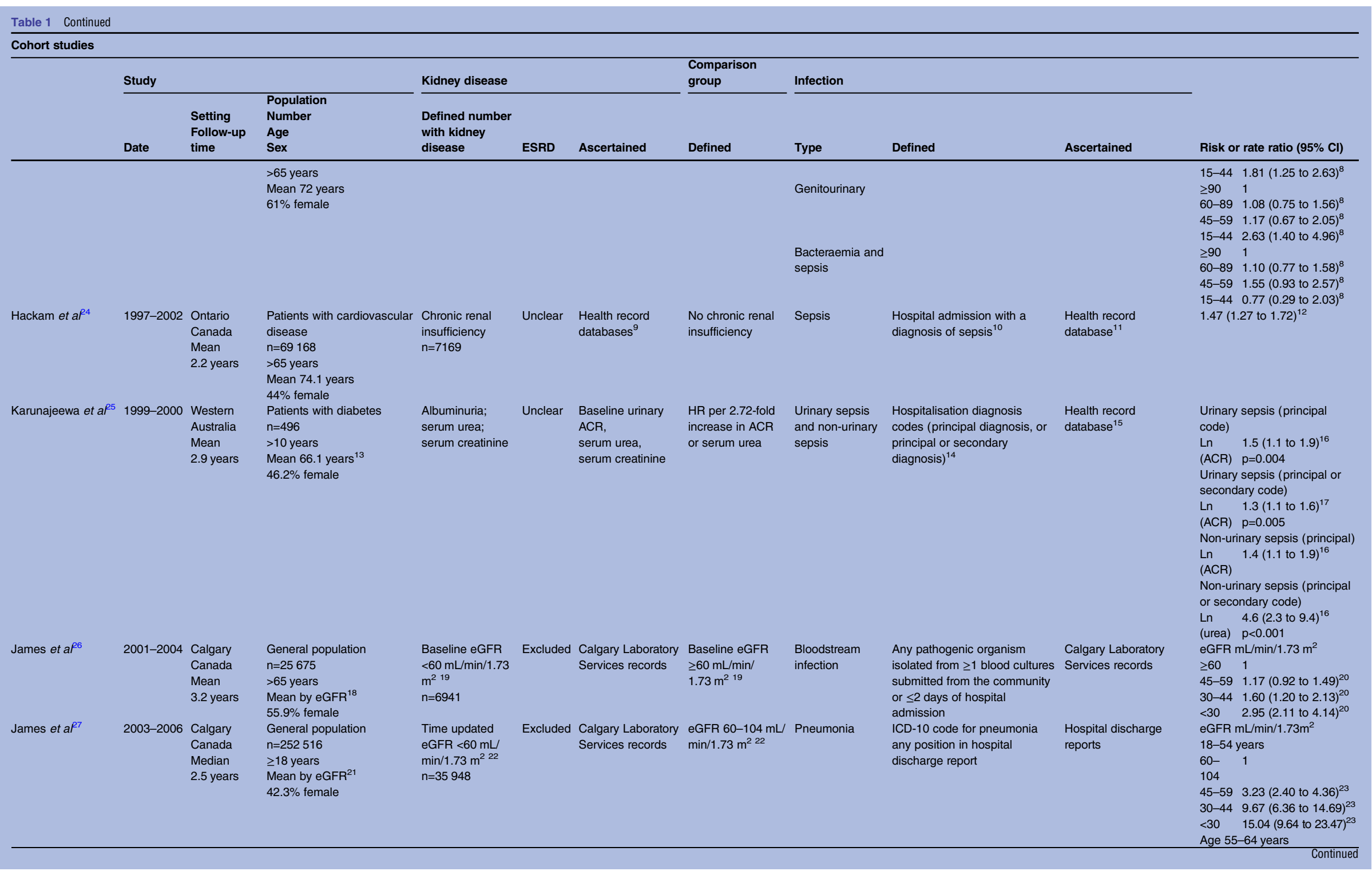




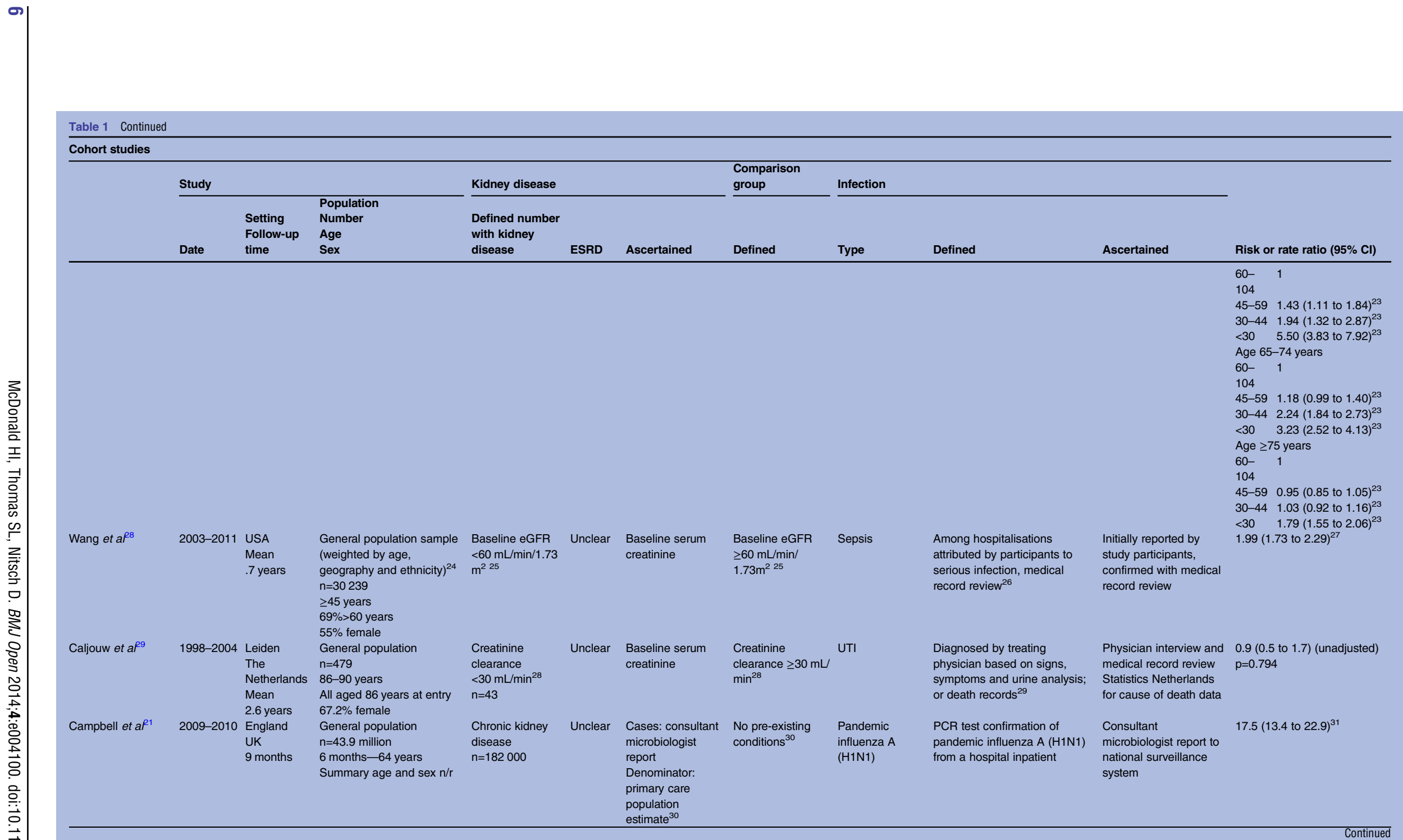




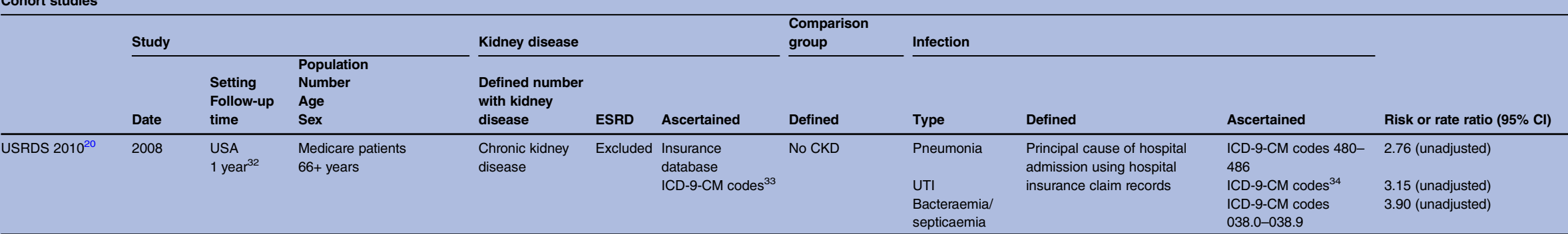

${ }^{1}$ Controls matched to cases on age at index data (within 1 year), sex, general practice and calendar time. Estimate adjusted for smoking status, Townsend deprivation score, use of influenza vaccine in previous 12 months, use of pneumococcal vaccine in previous 5 years, number of years of medical records data available in database, and comorbidities including: diabetes, chronic heart disease, chronic respiratory disease, asplenia, cerebrospinal shunt, chronic liver disease, sickle cell disease or coeliac disease, cochlear implant, HIV/AIDS, immunosuppression, stroke or transient ischaemic attack, rheumatoid arthritis, Parkinson's disease, multiple sclerosis, dementia, osteoporosis and any cancer.

${ }^{2}$ Center for American Indian Health surveillance system.

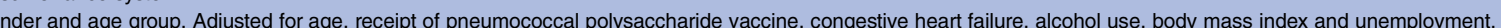

${ }^{4}$ Adjusted for age, non-English language spoken most at home, living in detached house, living alone, congestive heart failure, chronic obstructive pulmonary disease, dysphagia, functional status using Barthel Index, immunosuppressive medications, nutritional score, tobacco use (lifetime history and secondhand smoke), alcohol consumption and history of regular exposure to gases, fumes or chemicals at home or at work.

${ }^{5}$ Approximate numbers, read from bar graph in publication. No Cls available.

${ }^{6}$ Cohort selected for the Cardiovascular Health Study. Exclusion criteria included: inability to provide informed consent or communicate with the interviewer, institutionalisation, being homebound, receipt of hospice care, treatment with radiation or

chemotherapy for cancer or plans to move out of the community within 3 years.

${ }^{7}$ Serum cystatin $C$ measured by particle-enhanced immunonephelometric assay, and eGFR calculated using: eGFR=6.7×CysC -1.19

${ }^{8}$ Adjusted for age, sex, race, tobacco use, body mass index, diabetes mellitus, coronary heart disease, stroke, heart failure, cancer, chronic obstructive pulmonary disease, serum albumin, $\mathrm{C}$ reactive protein, interleukin-6.

${ }^{9} \mathrm{C}$ anadian Institute for Health Information Discharge Abstract database or Ontario Health Insurance Plan database.

${ }^{10}$ ICD-9 codes 0031,0362 and 038 0-038 9.

${ }_{11}^{11}$ Canadian Institute for Health Information Discharge Abstract database.

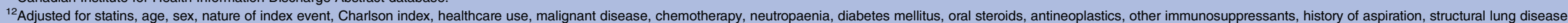
infection (respiratory, Gl, skin/soft tissue or other), recent trauma, transplant recipient, heart failure, stroke, chronic liver disease, chronic obstructive pulmonary disease, alcoholism, dementia, Parkinson's disease. Statin and non-statin users matched using propensity scoring for the above factors.

${ }^{13}$ Mean age among the 460 participants without asymptomatic bacteriuria, 66.1 years (SD 11.0): mean age among the 36 participants with asymptomatic bacteriuria, 67.7 years (SD 10.5).

${ }^{14}$ ICD-9 or ICD-10 codes for urinary sepsis were those encoding UTI, cystitis, pyelonephritis, orchitis, epididymitis and prostatitis; codes for non-urinary sepsis were those for sepsis, septicaemia and/or abscess.

${ }^{15}$ Western Australia Data Linkage System.

${ }^{16}$ Adjusted for presence of asymptomatic bacteriuria.

${ }^{17}$ Adjusted for presence of asymptomatic bacteriuria and age.

${ }^{18}$ Mean age + SD by eGFR. $>60: 74.4 \pm 6.5$ years. $45-59: 77.5+7.2$ years. $30-44: 79.3+7.4$ years. $<30: 78.6 \pm 7.4$ years.

${ }^{19}$ eGFR calculated using abbreviated Modification of Diet in Renal Disease Study equation (omitting ethnicity) from single outpatient serum creatinine result.

${ }^{20}$ Adjusted for age, sex, diabetes mellitus, comorbidity score and care in a CKD clinic.

${ }^{21}$ Mean age \pm SD by eGFR. $\geq 105: 38.7 \pm 14.6 .60-104: 50.9 \pm 15.4$. 45-59: $67.0 \pm 14.1 .30-44: 74.5 \pm 12.9 .<30: 73.3 \pm 15.2$

${ }^{22}$ eGFR calculated using abbreviated Modification of Diet in Renal Disease Study equation (omitting ethnicity) from most recent outpatient serum creatinine result.

${ }^{23}$ Adjusted for age, sex, socioeconomic status, ethnicity, diabetes mellitus, Charlson comorbidity score.

${ }^{24}$ Cohort selected for the Reasons for Geographic and Racial Differences in Stroke (REGARDS) study. Population weighted by age, ethnicity and geography according to local stroke incidence rates.

${ }^{25}$ eGFR calculated using the CKD-EPI equation.

${ }^{26} \mathrm{Medical}$ record review confirming (1) serious infection as the major reason for admission and (2) $\geq 2$ of heart rate $>90 \mathrm{bpm}$, temperature $>38.3^{\circ} \mathrm{C}$ or $<36^{\circ} \mathrm{C}$, tachypnoea $>20$ breaths/min or leucocytosis

${ }^{27}$ Adjusted for age, sex, race, education, income, geographical region, alcohol use and smoking status.

${ }^{20}$ Creatinine clearance calculated from serum creatinine concentration and weight using the Cockcroft-Gault formula.

${ }^{20}$ Cause of death recorded as UTI (ICD-10 code N39.0)/

${ }^{30}$ Department of Health-Health Protection Agency influenza vaccine uptake primary care monitoring system data.

${ }^{31}$ Adjusted for age.

${ }^{32}$ Smoothed estimate: models include data from the stated year and the 2 years preceding it, applying weights of $1,1 / 4$ and $1 / 8$ with increasing distance in time.

ICD-9-CM diagnosis codes recorded in insurance claims during the preceding year: 585.1-585.5 (chronic kidney disease stages 1-5); or 585.6 with no ESRD 2728 form or other indication of ESRD.

PAPrincipal hospital admission ICD-9-CM codes: 590-590.9, 595-595.4, 597-597.89, 598, 599.0, 601-601.9, 604-604.9, 607.1-2, 608.0, 608.4, 616.1, 616.3-4 and 616.8.

System; UTI, urinary tract infection. 
Definitions of kidney disease included medical diagnoses of chronic renal disease, elevated creatinine levels, impaired creatinine clearance and structural abnormalities of the kidney. Five studies excluded patients with ESRD, and one specified the number included, but for the remaining eight studies it was unclear how many of the included patients received renal replacement therapy (table 1).

Three studies recorded infections diagnosed in primary care or outpatients, ${ }^{16} 1929$ two recorded infections identified from a positive culture result, ${ }^{17}{ }^{26}$ one included infections diagnosed in the emergency department, ${ }^{18}$ seven required hospital admission for infection $^{5} 21$ 23-25 2728 and for one study the definition and severity of infection was unclear. ${ }^{22}$

For two studies, the results extracted had no CI or SE and these could not be calculated from the reported data. From the remaining 12 studies, 17 independent effect estimates with SEs were available for meta-analysis, among which UTI was the outcome in three estimates.

For all infections, there was strong evidence of considerable heterogeneity (Cochran's $Q$ statistic $\mathrm{p}<0.001$, $\left.\mathrm{I}^{2}=96.5 \%\right)$. This persisted when estimates for UTIs were excluded $\left(\mathrm{p}<0.001, \mathrm{I}^{2}=97.2 \%\right)$, when considering LRTIs alone $\left(p<0.001, \mathrm{I}^{2}=98.2 \%\right)$, when limited to cohort studies $\left(\mathrm{p}<0.001, \mathrm{I}^{2}=97.3 \%\right)$, and when stratified by exclusion of patients with ESRD (ESRD excluded, $\mathrm{p}<0.001, \mathrm{I}^{2}=88.9 \%$ : ESRD not excluded $\left.\mathrm{p}<0.001, \mathrm{I}^{2}=97.2 \%\right)$. Owing to this heterogeneity, meta-analysis was not performed.

All results are displayed in the Forest plot (figure 2). Despite the quantitative heterogeneity, the results were qualitatively similar: all estimates were compatible with a positive association between kidney disease and infection. The four studies which compared different stages of CKD found a graded association of increased risk of infection with more severe CKD. All four of these studies excluded patients with ESRD. ${ }^{22} 232627$ One study found that the effect of CKD on infection risk was modified by age, with a declining effect of CKD on infection risk as age increased. ${ }^{27}$ This effect was consistent with the lower effect of CKD on UTI incidence found among 86-90 year-olds $(0.90,95 \%$ CI 0.50 to 1.77) compared with an adult study population with a mean age of 66 years $(1.50,95 \%$ CI 1.10 to 1.90$) .^{25} 29$

The funnel plot was sparsely populated, with widely scattered effect estimates, and provides no clear evidence for or against publication bias (see online supplementary figure $\mathrm{S} 1$ ).

Study quality was variable. Relying on routine medical diagnosis introduced a potential source of misclassification of kidney disease status for seven studies. ${ }^{5}$ 16-19 2124 There was variable adjustment for confounding, from unadjusted crude estimates to estimates adjusted for a range of comorbidities, demographic and socioeconomic factors. Six studies did not meet this review's minimal requirements. ${ }^{19} 2122252829$ The summarised results are displayed in table 2 , and the full quality assessment is in online supplementary table S5.

\section{DISCUSSION}

Our comprehensive search strategy identified 14 studies describing an association between kidney disease and acute community-acquired infection. Although betweenstudy heterogeneity precluded meta-analysis, all studies were consistent with a positive direction of association. Four studies which reported estimates on more than one category of kidney disease found a graded association in which risk of infection increased with greater severity of CKD. These four studies excluded patients with ESRD, and three were at low risk of bias in all categories of quality assessment. ${ }^{22} 232627$

To the best of our knowledge, this is the first review to address this research question systematically. We used a sensitive search strategy, with a broad definition of kidney disease, for a thorough and inclusive search. The results are consistent with the conclusion of previous narrative reviews: that an association between CKD and infection incidence is likely, but that there is a paucity of evidence. $^{10-12}$

Heterogeneity between the studies precluded a meta-analysis of results. Variable study designs and biases may have contributed to the heterogeneity: for example, the four case-control studies calculated ORs, which may differ from equivalent rate ratios for common infections. ${ }^{16-19}$ Failure to control the confounding effects of age, sex and diabetes would be likely to result in overestimation of the effect of CKD on infection. Nondifferential misclassification of kidney disease status in studies which relied on routine medical diagnosis would be expected to underestimate the effect of CKD on infection risk. In general, the risk of ascertainment bias from increased monitoring for infection among patients with CKD is probably low, although one study assessed risk factors for hospitalisation with influenza during an influenza pandemic, in which context patients with influenza-like symptoms may have been more likely to be tested for influenza $\mathrm{A}(\mathrm{H} 1 \mathrm{~N} 1)$ if they also had CKD. ${ }^{21}$

The heterogeneity may reflect true differences in effect size between the studies.

First, the studies considered a range of outcomes. CKD may have a different effect on the incidence of different infections. For all but three studies, detection of infection required either hospital attendance for the infection or a positive blood culture. CKD may affect severity of infection, as an alternative, or in addition to any effect on infection incidence. CKD may also increase the probability of hospital admission for management of a moderately severe infection. Either would result in a larger effect of CKD on the risk of severe infectious outcomes (such as hospitalisation for sepsis) than on less severe infections (such as community-diagnosed LRTI), and could result in the graded association we observed, with increasing hospitalisation for patients with more severe stages of CKD.

Second, the studies included a variety of definitions of kidney disease. For example, proteinuria (and renal loss of complement) may represent a separate mechanism 
Relative risk

$(95 \% \mathrm{Cl})$

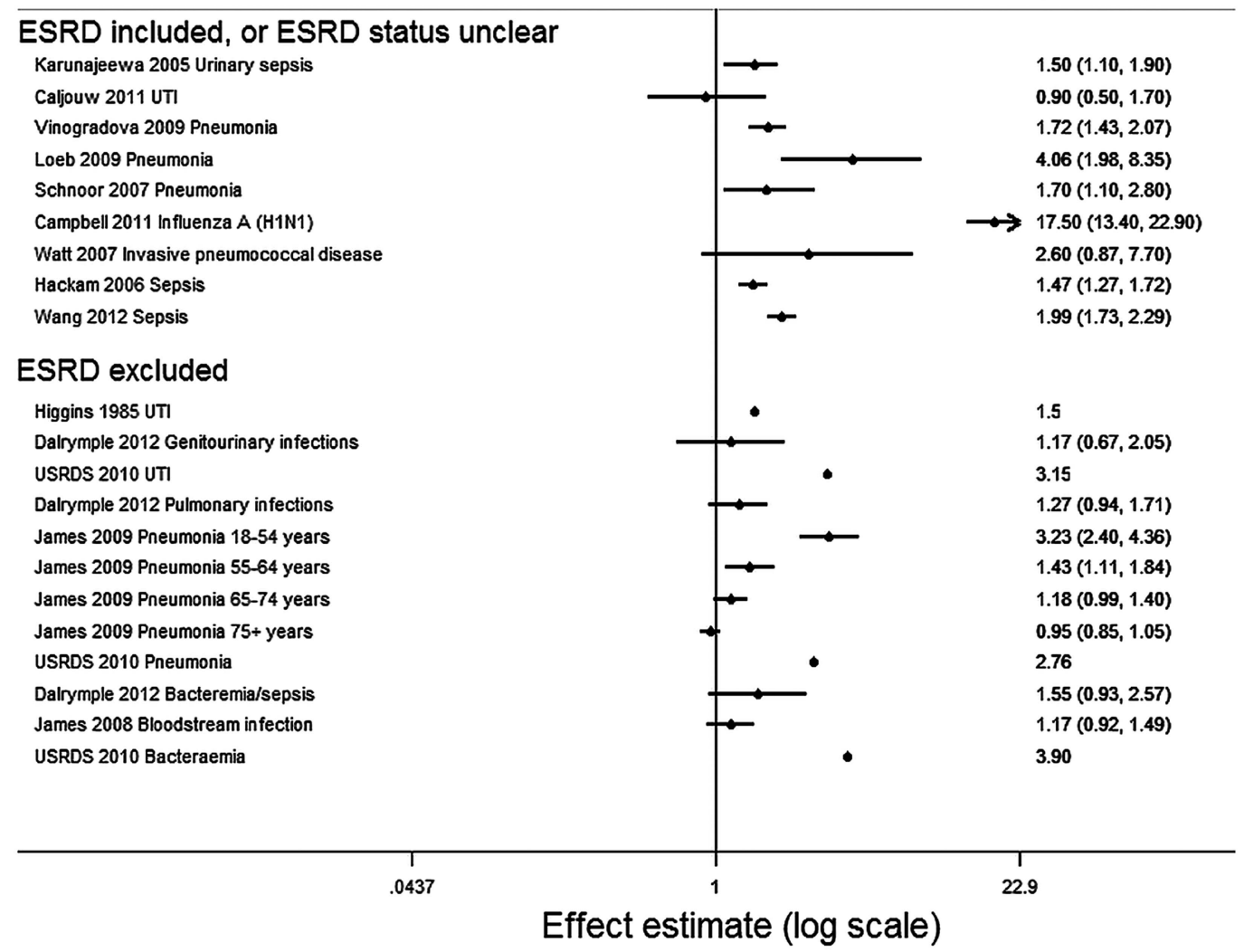

Figure 2 Forest plot of all estimates of the association of chronic kidney disease with infection ( $\mathrm{n}=17)$ from all 14 studies identified. The estimates from Higgins 1985 and USRDS 2010 did not include SEs. Dalrymple 2012: presented estimates compare eGFR 45-59 with eGFR $\geq 90 \mathrm{~mL} / \mathrm{min} / 1.73 \mathrm{~m}^{2}$; James 2009: presented estimates compare eGFR 45-59 with eGFR 60$104 \mathrm{~mL} / \mathrm{min} / 1.73 \mathrm{~m}^{2}$; James 2008: presented estimates compare eGFR $45-59$ with eGFR $\geq 60 \mathrm{~mL} / \mathrm{min} / 1.73 \mathrm{~m}^{2}$. eGFR, estimated glomerular filtration rate; ESRD, end-stage renal disease; USRDS, US Renal Data System; UTI, urinary tract infection.

for risk of infection than uraemia. For the nine studies which did not exclude patients with ESRD, it is unclear to what extent the results reflect the effect of treatments associated with dialysis, such as vascular or peritoneal access for dialysis, on infection incidence.

Third, the association of CKD with infection may be modified by age. James et al observed a weaker association of CKD with hospitalisation for pneumonia as age increased. They suggested that such an observation could be explained by a lower baseline rate of hospitalisation for pneumonia among younger adults, the natural decline in renal function by age, and inaccuracy in the estimation of renal function using the Modification of Diet in Renal Disease (MDRD) study equation in older populations. ${ }^{27}$ As their study population included only adults who had had a creatinine test result, reasons for testing creatinine could also be relevant confounders. As age increases, more comorbidities accrue which require creatinine tests to guide therapy. Hence, younger people who receive a creatinine test may be at an unusually high risk for infections and CKD due to the reasons associated with getting a creatinine test. A real age-dependency of the CKD-infection association would be consistent with the lower effect of CKD on UTI incidence found among 86-90-year-olds (0.90, $95 \%$ CI 0.50 to 1.77 ) compared with an adult study population with a mean age of 66 years $(1.50,95 \%$ CI 1.10 to 1.90$)$. However, it may be that the study among the older adults measured a less severe outcome, and CKD may be associated with other factors that eventually lead to hospitalisation for UTI. ${ }^{25} 29$

CKD was not a component of the primary study question for nine of the 14 studies; thus, there is a risk that this association may have been reported and published only when CKD was found to be a risk factor for infection or an important confounder of another relationship. This would result in selective reporting bias, with a subsequent overestimation of the association of CKD with infection risk. This bias would be expected to affect smaller studies to a greater extent, and a funnel plot might show an asymmetry of relative risk estimates about the central pooled estimate among smaller studies. The sparsely populated funnel plot (see online supplementary figure S1) provides no clear evidence for or against selective reporting bias, but some evidence of selective reporting bias comes from within the individual studies. For example, the crude HR for the association of creatinine clearance with UTI incidence is reported in 


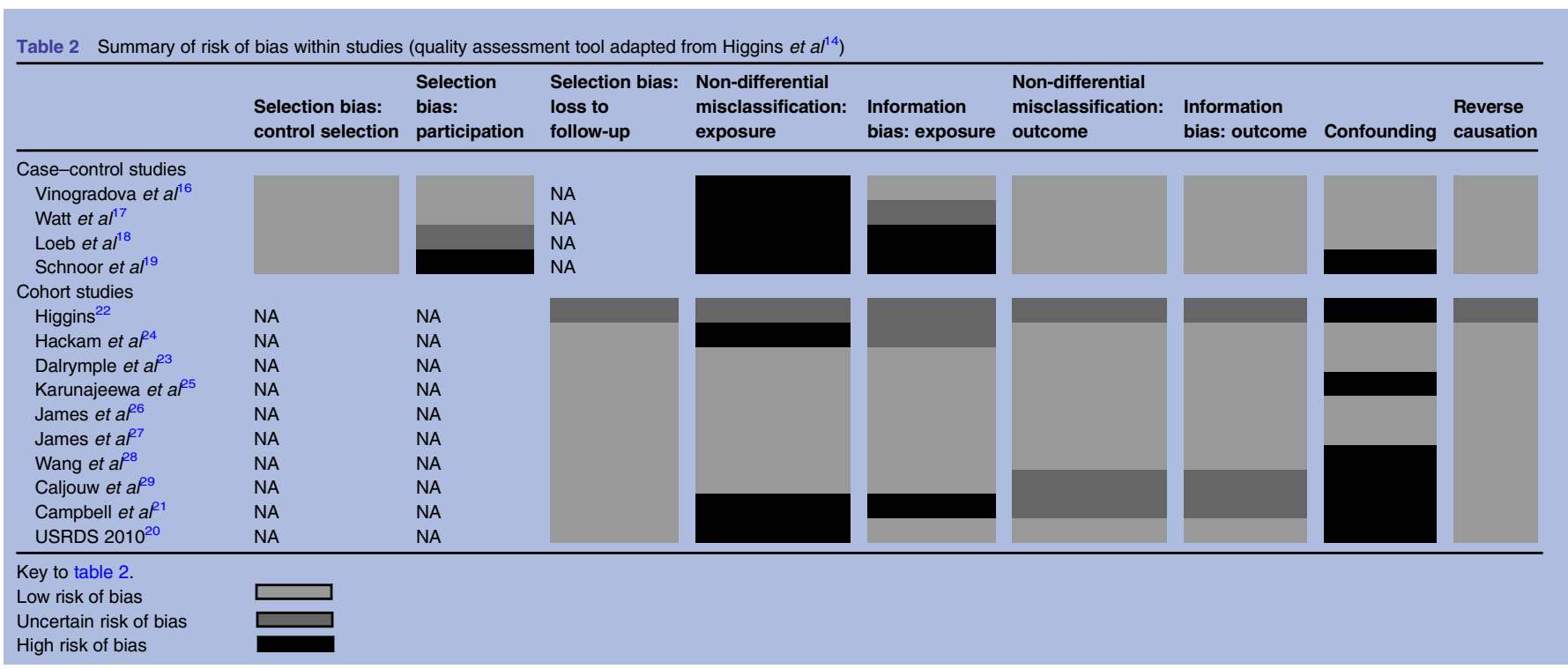

Caljouw et $a l^{29}(0.9,95 \%$ CI 0.5 to 1.7$)$, but as creatinine clearance was not found to be significant in the multivariable model, the adjusted association is not reported.

The overlap in the study populations of the two large cohort studies based in Calgary, Canada could result in more similar estimates than if the study populations were independent. ${ }^{2627}$ Outcomes in the two studies are likely to be correlated with each other: hospitalisation with pneumonia could cause a positive blood culture, which would result in one infection being included as an outcome in both studies. This is unlikely to have a large effect, particularly in the qualitative assessment of the combined evidence, as the potential overlap of person-time is limited.

Although we excluded study populations routinely treated with specialist medication (unless for kidney disease), some study populations may have been at higher risk of infection than the general population, and this may have affected the relationship of CKD to infection. For example, the cohort of patients admitted for an acute cardiovascular event or an arterial revascularisation procedure will have had a higher prevalence of comorbidities (such as diabetes) than the general population and excluded patients with severe comorbidities who did not survive an acute cardiovascular event, or who were not fit enough to undergo the procedure. ${ }^{24}$ Each of the selected study populations limits the generalisability of the individual study result, but the qualitatively similar findings across the variety of study populations, and their qualitative consistency with the studies based among the general population, 5161921232829 support a positive association between CKD and infection risk in a variety of study populations.

A few large, high-quality studies which excluded patients with ESRD have found a graded association between predialysis CKD and risk of hospitalisation with infection. All studies identified in this review were compatible with a positive association of CKD with increased infection risk. There are little data available on the association of CKD with infection incidence using less severe outcome measures than hospitalisation, and it is not possible in most studies to distinguish an effect on susceptibility to infection from an effect on the severity of infection.

The potential age-dependency of the relationship between CKD and infection is intriguing and needs further research. Also, there is currently no evidence on the relationship between proteinuria and infection incidence independent of the glomerular filtration rate. Future studies should identify infections in the community in addition to hospitalisations for infection, characterise the association of proteinuria adjusted for the glomerular filtration rate, explore the age-dependency of the association and assess vaccine efficacy among older people with CKD.

Contributors All authors designed the study strategy including the search terms, inclusion and exclusion criteria. HIM performed the search, study selection and data extraction. DN screened the randomly selected sample of 100 abstracts. All authors agreed on the quality assessment of included papers and interpretation of results by discussion. HIM drafted the article, which DN and SLT revised. All authors approved the final version of the manuscript.

Funding This research received no specific grant from any funding agency in the public, commercial or not-for-profit sectors.

Competing interests This report is independent research arising from a Career Development Fellowship supported by the National Institute for Health Research, awarded to SLT (grant number CDF 2010-03-32). HIM is funded by a Kidney Research UK studentship, grant reference ST2/2011.

Provenance and peer review Not commissioned; externally peer reviewed.

Data sharing statement All data, including full search terms and eligibility criteria, are available either in the article or as online supplementary material submitted with this manuscript.

Open Access This is an Open Access article distributed in accordance with the Creative Commons Attribution Non Commercial (CC BY-NC 3.0) license, which permits others to distribute, remix, adapt, build upon this work noncommercially, and license their derivative works on different terms, provided 
the original work is properly cited and the use is non-commercial. See: http:// creativecommons.org/licenses/by-nc/3.0/

\section{REFERENCES}

1. Coresh J, Selvin E, Stevens LA, et al. Prevalence of chronic kidney disease in the United States. JAMA 2007;298:2038-47.

2. Collins AJ, Foley R, Herzog C, et al. United States Renal Data System 2007 Annual Data Report Abstract. Am J Kidney Dis 2008:51:A6-7.

3. Sarnak MJ, Jaber BL. Mortality caused by sepsis in patients with end-stage renal disease compared with the general population. Kidney Int 2000;58:1758-64.

4. Sarnak MJ, Jaber BL. Pulmonary infectious mortality among patients with end-stage renal disease. Chest 2001;120:1883-7.

5. US Renal Data System 2011 Annual Data Report. Morbidity and mortality in patients with chronic kidney disease. Am J Kidney Dis 2012;59:e59-68.

6. Naqvi SB, Collins AJ. Infectious complications in chronic kidney disease. Adv Chronic Kidney Dis 2006;13:199-204.

7. Viasus D, Garcia-Vidal C, Cruzado JM, et al. Epidemiology, clinical features and outcomes of pneumonia in patients with chronic kidney disease. Nephrol Dial Transplant 2011;26:2899-906.

8. Kausz AT, Gilbertson DT. Overview of vaccination in chronic kidney disease. Adv Chronic Kidney Dis 2006;13:209-14.

9. Allon M, Depner TA, Radeva M, et al. Impact of dialysis dose and membrane on infection-related hospitalization and death: results of the HEMO Study. J Am Soc Nephrol 2003;14:1863-70.

10. Dalrymple LS, Go AS. Epidemiology of acute infections among patients with chronic kidney disease. Clin J Am Soc Nephrol 2008:3:1487-93.

11. Foley RN. Infections in patients with chronic kidney disease. Infect Dis Clin North Am 2007;21:659-72, viii.

12. Foley RN. Infections and cardiovascular disease in patients with chronic kidney disease. Adv Chronic Kidney Dis 2006;13:205-8.

13. The World Bank. Country and lending groups. 2012 [6 Jun 2013]. http://data.worldbank.org/about/country-classifications/ country-and-lending-groups

14. Higgins JP, Altman DG, Gotzsche PC, et al. The Cochrane Collaboration's tool for assessing risk of bias in randomised trials. BMJ 2011;343:d5928.
15. Higgins JP, Thompson SG, Deeks JJ, et al. Measuring inconsistency in meta-analyses. BMJ 2003;327:557-60.

16. Vinogradova Y, Hippisley-Cox J, Coupland C. Identification of new risk factors for pneumonia: population-based case-control study. Br J Gen Pract 2009;59:e329-38.

17. Watt JP, O'Brien KL, Benin AL, et al. Risk factors for invasive pneumococcal disease among Navajo adults. Am J Epidemiol 2007;166:1080-7.

18. Loeb M, Neupane B, Walter SD, et al. Environmental risk factors for community-acquired pneumonia hospitalization in older adults. J Am Geriatr Soc 2009;57:1036-40.

19. Schnoor M, Klante T, Beckmann M, et al. Risk factors for community-acquired pneumonia in German adults: the impact of children in the household. Epidemiol Infect 2007;135:1389-97.

20. Collins AJ, Foley RN, Herzog C, et al. US Renal Data System 2010 Annual Data Report. Am J Kidney Dis 2011;57(1 Suppl 1):A8, e1-526.

21. Campbell CNJ, Mytton OT, McLean EM, et al. Hospitalization in two waves of pandemic influenza $\mathrm{A}(\mathrm{H} 1 \mathrm{~N} 1)$ in England. Epidemiol Infect 2011;139:1560-9.

22. Higgins RM. Infections in a renal unit. $Q J$ Med 1989;70:41-51.

23. Dalrymple LS, Katz R, Kestenbaum B, et al. The risk of infection-related hospitalization with decreased kidney function. Am J Kidney Dis 2012;59:356-63.

24. Hackam DG, Mamdani M, Li $P$, et al. Statins and sepsis in patients with cardiovascular disease: a population-based cohort analysis. Lancet 2006;367:413-18.

25. Karunajeewa H, McGechie D, Stuccio G, et al. Asymptomatic bacteriuria as a predictor of subsequent hospitalisation with urinary tract infection in diabetic adults: the Fremantle Diabetes Study. Diabetologia 2005;48:1288-91.

26. James MT, Laupland KB, Tonelli M, et al. Risk of bloodstream infection in patients with chronic kidney disease not treated with dialysis. Arch Intern Med 2008;168:2333-9.

27. James MT, Quan H, Tonelli M, et al. CKD and risk of hospitalization and death with pneumonia. Am J Kidney Dis 2009;54:24-32.

28. Wang HE, Shapiro NI, Griffin R, et al. Chronic medical conditions and risk of sepsis. PLOS ONE 2012;7:e48307.

29. Caljouw MA, den Elzen WP, Cools HJ, et al. Predictive factors of urinary tract infections among the oldest old in the general population. A population-based prospective follow-up study. BMC Med 2011;9:57. 